\title{
Fraternité en éducation : le point de vue de Jean- Paul Delahaye
}

\section{Agnès Perrin-Doucey and Jean-Paul Delahaye}

\section{(2) OpenEdition \\ Journals}

Electronic version

URL: http://journals.openedition.org/trema/5710

DOI: 10.4000/trema.5710

ISSN: 2107-0997

Publisher

Faculté d'Éducation de l'université de Montpellier

\section{Electronic reference}

Agnès Perrin-Doucey and Jean-Paul Delahaye, «Fraternité en éducation : le point de vue de Jean-Paul Delahaye », Tréma [Online], 53 | 2020, Online since 01 March 2020, connection on 03 March 2020. URL : http://journals.openedition.org/trema/5710; DOI : 10.4000/trema.5710

This text was automatically generated on 3 March 2020

Trema 


\title{
Fraternité en éducation : le point de vue de Jean-Paul Delahaye
}

\author{
Agnès Perrin-Doucey and Jean-Paul Delahaye
}

\section{Introduction}

1 Pour penser l'idée de Fraternité en éducation comme point d'ancrage d'une éducation à la fraternité, il est indispensable d'en cerner les contours institutionnels, politiques et sociétaux contemporains. Certes, l'intentionnalité politique, définie par le cadre axiologique et législatif républicain, porte l'idée d'une école gratuite, ouverte à tous, donc d'une école favorisant l'égalité de tous les citoyens devant l'instruction. Pourtant, le passage d'une égalité en droits vers une égalité concrétisée dans les faits reste pour beaucoup de l'ordre d'un idéal inaccessible, alors même que la devise républicaine en porte la promesse. Si l'idée de fraternité, au sens républicain du terme, a été introduite dans la devise, c'est bien pour accompagner le passage des droits aux faits et favoriser un engagement citoyen respectueux et soucieux de l'altérité. La mise en œuvre d'une école qui offrirait une réelle égalité des chances était l'un des piliers de ce projet de société. Mais au $21^{\text {ème }}$ siècle, l'éducation nationale semble ne plus atteindre cet objectif. L'Organisation de Coopération et de Développement Economiques (OCDE) dans ses rapports internationaux l'atteste de façon récurrente: la France peine à assurer l'égalité des chances à ses citoyens. L'Observatoire National de la Pauvreté et de l'Exclusion Sociale ${ }^{1}$, commentant une étude réalisée par le think tank France Stratégie sur la période 2011-2014, affirme que les facteurs déterminants cette inégalité sont avant éducatifs ${ }^{2}$. Nous postulons que pour rétablir dans les faits une plus grande égalité des chances, il est nécessaire de prendre en compte l'idée de fraternité et de l'installer au cœur des politiques publiques d'éducation, mais aussi au centre de l'action éducative. Bien évidemment, la volonté et l'affirmation de grands principes axiologiques ne suffisent pas, il faut surtout penser l'action permettant de mettre la fraternité (au sens de solidarité citoyenne et républicaine) au cœur de l'éducation. Quelles actions, quels engagements politiques et citoyens ce projet implique-t-il ? Si le présent ouvrage vise à offrir des réponses philosophiques mais aussi didactiques et 
pédagogiques à cette question fondamentale, nous avons aussi souhaité recueillir le point de vue de Jean-Paul Delahaye, aujourd'hui Inspecteur Général de l'Education Nationale honoraire. En effet, son parcours professionnel, comme ses engagements citoyens ont fait de lieu un observateur, un analyste mais aussi un acteur de premier plan au service de l'éducation.

2 Présenter la biographie professionnelle et citoyenne de Jean-Paul Delahaye n'est pas chose aisée, tant elle est riche. Cependant, comme nous allons le montrer, elle résume, à elle seule, ce projet républicain d'élévation sociale et confère ainsi à notre échange et surtout à son témoignage comme à son analyse, une légitimité puisée tant dans le réalisme de l'expérience et de l'action, que dans la puissance symbolique de son parcours. En effet, son parcours lui a permis de se confronter à de nombreuses missions qui traversent l'essentiel des corps stratégiques au service de l'enseignement: enseignant, cadre de l'éducation tant en formation que dans les services académiques, haut fonctionnaire des services d'éducation. De 1973 à 1982, notre interlocuteur débute sa carrière comme professeur d'histoire-géographie, de 1982 à 1986, il devient Inspecteur de l'éducation nationale pour le premier degré, puis Directeur d'Ecole Normale (1986-1990), basculant ainsi de l'enseignement à l'inspection, de l'inspection à la formation. De 1990 à 2001, sa carrière prend un tour nouveau, puisqu'il accède aux fonctions d'Inspecteur d'académie-adjoint, puis d'Inspecteur d'académie de plein exercice. Il exerce alors successivement dans le Bas-Rhin (adjoint), la Haute Saône, la Côte d'Or et la Seine-Saint-Denis rencontrant ainsi des réalités professionnelles fort différentes. En 2001, fort de cette riche expérience de terrain, il est nommé Inspecteur Général de l'Education Nationale, en activité jusqu'en 2016 (honoraire à ce jour), et exerce la mission de doyen du groupe «Établissements et vie scolaire» de 2002 à 2006. Docteur en sciences de l'éducation, spécialiste de l'histoire de l'éducation et historien des politiques scolaires, il assure, dans le même temps (2003-2011), une mission de professeur associé à l'Université Paris 5 Sorbonne.

3 Ce parcours professionnel le conduit aussi à l'action politique : il devient tour à tour, conseiller du ministre Jack Lang (2001-2002) et conseiller spécial du ministre de l'éducation nationale Vincent Peillon (2012). De 2012 à 2014, il accède au poste de Directeur général de l'enseignement scolaire. Il participe alors à la préparation et à la première mise en œuvre de la loi de refondation de l'école de la république du 8 juillet 2013, ainsi qu'à l'écriture et la mise en place de la Charte de la laïcité à l'école. Enfin, en 2015, il remet un rapport « Grande pauvreté et réussite scolaire, le choix de la solidarité pour la réussite de tous $»^{3}$ qui ne laisse personne indifférent tant les situations décrites sont criantes de réalisme.

4 Enfin, l'homme est aussi un citoyen engagé d'une part à la Ligue de l'enseignement dont il est administrateur, d'autre part au Comité national d'action laïque qu'il a présidé en 2018, coordonnant l'enquête «La laïcité à l'école, les enseignants ont la parole».

\section{Interview}

\section{Faire tenir debout notre devise républicaine}

5 A. Perrin-Doucey : Pour vous, qu'est-ce que la fraternité républicaine? 
6 J.-P. Delahaye : La fraternité c'est ce qui fait se tenir debout notre devise républicaine et lui donne tout son sens: pas de liberté ni d'égalité possible entre les citoyens sans fraternité. Nos valeurs forment un tout. C'est Antoine de Saint-Exupéry qui disait « Une démocratie doit être une fraternité, sinon c'est une imposture $»^{4}$. La fraternité c'est ce qui rassemble autour d'un projet commun des citoyens différents mais qui sont des semblables d'égale dignité. Pour se rassembler, nul besoin de se ressembler mais il faut qu'une solidarité en actes permette à chacun d'apporter sa contribution à la vie collective. C'est en ce sens que la fraternité permet d'une part de lutter contre les rejets, les replis communautaires et identitaires et d'autre part de combattre les inégalités sociales qui sont un déni de nos valeurs.

En tout état de cause, l'honneur d'une société se mesure à la place qu'elle fait à ceux qui sont, à un moment donné, en situation de fragilité.

7 A. Perrin-Doucey : Où en est-on aujourd'hui en matière de fraternité en éducation?

\section{Un fonctionnement conforme aux valeurs}

8 J.-P. Delahaye: Le système éducatif dans son ensemble, comme les unités d'enseignement, écoles et établissements, fonctionnent le plus souvent et fort heureusement conformément aux valeurs et aux principes affichés. Notre école n'a pas à rougir de son action. Il faut le rappeler en ces temps où le déclinisme est porteur. Je pense évidemment à tous ceux qui ne cessent de répéter que « c'était mieux avant ». En quarante ans, et j'en ai vu la manifestation dans tous les postes que j'ai occupés dans ma carrière, notre école s'est en effet transformée et a transformé la France. Prenons une illustration de ces progrès considérables. Quel plus bel exemple d'action collective fraternelle que la politique conduite dans notre pays qui a fait que le nombre des sorties annuelles sans qualification qui restait bloqué à 140000 en 2010 vient de passer après dix ans d'efforts à moins de 90000 . C'est le fruit d'une mobilisation générale pour mieux connaître les décrocheurs, mieux prévenir le décrochage en mobilisant l'école et ses partenaires. J'allais dire comme Abdenour Bidar que cette action publique est le fruit d'une « fraternité générale » au-delà des alternances politiques.

\section{« Des valeurs qui parfois servent d'alibi ou de faux-nés »...}

Mais, une fois que l'on a dit cela, la lucidité nous oblige à poser cette question, pas seulement à l'école mais à l'ensemble de la société : comment rendre compatibles avec les valeurs républicaines, et donc la fraternité, avec de tels écarts de réussite entre nos élèves et nos établissements scolaires? Comment rendre compatible avec ces mêmes valeurs républicaines l'existence simultanée d'établissements scolaires en grande difficulté et d'établissements scolaires, pas seulement privés mais aussi publics, réservés à une élite sociale et à quelques boursiers méritants? Comment proclamer sans vergogne nos valeurs et supporter l'échec scolaire précoce et cumulatif de $15 \%$ environ des jeunes, essentiellement issus des familles les plus défavorisées, échec qui atteste la permanence de l'effet des inégalités sociales sur les parcours scolaires et met gravement en cause l'idéal du système éducatif d'assurer l'égalité des enfants. Et comme chacun le sait, le poids de l'origine sociale sur les destins scolaires est nettement supérieur chez nous que dans la plupart des pays de l'OCDE. Quand on observe que $90 \%$ des enfants de cadres et d'enseignants ont le baccalauréat sans 
problème sept ans après leur entrée en sixième et que ce n'est le cas que pour $40 \%$ des enfants d'ouvriers, on voit bien qu'il n'y a guère plus de progrès à espérer pour certains mais que les marges de progression pour les autres sont énormes.

Le problème, c'est quand on fait de la mise en avant des valeurs, une sorte d'alibi ou de faux-nez, pour masquer des agissements parfaitement contraires à nos valeurs.

A. Perrin-Doucey : Pourriez-vous exemplifier ce propos?

J.-P. Delahaye : Par exemple, quelle fraternité réelle existe-t-il dans nos collèges quand une étude du CNESCO de 2015 montre qu'en classe de troisième, $45 \%$ des collèges pratiquent une ségrégation scolaire active et $25 \%$ des formes de séparatisme social en mettant en place des options ou des parcours spécifiques qui permettent à certains citoyens de protéger en quelque sorte leurs enfants de la fréquentation de tous les autres ${ }^{5}$. Comment prétendre à une citoyenneté partagée par la jeunesse quand une partie d'entre elle se rend très vite compte que le collège qui l'accueille n'a pas été pensé pour tous mais uniquement pour les élèves qui sont programmés pour la voie générale du lycée. Comment expliquer que, chaque fois que l'on veut élargir la base sociale de la réussite on soit immédiatement accusé de nivellement par le bas par ceux qui veulent garder les positions acquises par leur classe sociale ? Et que dire enfin d'un lycée qui sélectionne ses élèves, qui refoule ses redoublants ou qui réoriente à tout va les élèves qui lui feraient perdre des places dans son rang de classement?

\section{«En France, on ne scolarise pas ensemble »}

Peut-il exister une citoyenneté partagée, une fraternité, sans mixité sociale et scolaire c'est-à-dire si ceux qui bénéficient d'un système élitiste, qui n'est pas de l'élitisme républicain mais qui est en réalité un élitisme social, continuent à vouloir isoler leurs enfants des enfants du peuple?

n France, qui prétend être le pays du "vivre ensemble » et de la fraternité, on ne scolarise pas ensemble. La partie de la population dont les enfants réussissent si bien dans une école qui procède par élimination s'est réservé de fait la voie générale. Elle valorise, dans les discours, l'apprentissage et l'enseignement professionnel, mais n'en fait pas une voie de réussite pour ses propres enfants. Elle y oriente les enfants des milieux populaires, ce qui a l'avantage de protéger ses enfants du contact des enfants des autres. Le système éducatif peut-il faire mieux quand une partie de la population semble faire sécession ${ }^{6}$ ?

A. Perrin-Doucey : Le problème est-il si important que vous semblez le dire ?

J.-P. Delahaye : Ne sous-estimons pas le problème. Ces pratiques placent en effet notre école publique en position délicate, une position qui, en quelque sorte, cristallise toutes les insatisfactions, dans la mesure où l'école publique agit en tant que représentant d'une République qui oublie parfois que la devise républicaine est un tout et qu'il est illusoire de penser faire vivre la liberté et l'égalité si on oublie la fraternité.

Le meilleur enseignement possible concernant les valeurs ne sera efficace que si la réalité sociale n'apporte pas de démenti à ce qui est enseigné. On demande à l'école de former des citoyens en mesure de comprendre la nécessité de l'intérêt général, or la société ambiante pousse toujours plus loin le culte de l'individualisme et la défense des intérêts particuliers. Comme le dit Régis Debray à la première ligne de son ouvrage, $L e$ moment Fraternité, « l'individu est tout et le tout n'est plus rien »". 

refusant la venue dans leur quartier de quelques pauvres que certains ont même osé qualifier de "nuisances ». Comment oser utiliser cette expression de "nuisances » pour qualifier les pauvres? Comment des êtres humains peuvent-ils parler ainsi d'autres êtres humains dans notre pays, aujourd'hui? Quelle fraternité, quelle citoyenneté en partage dans ces territoires de la République que je considère comme des territoires perdus de la république d'une certaine manière ${ }^{9}$, territoires où la mixité sociale est vécue comme un drame épouvantable par ceux qui se sont barricadés dans ce qu'on peut appeler des ghettos de riches? C'est dans ces territoires qui se sont protégés des autres qu'il faudrait conduire des actions d'éducation ou de rééducation à la fraternité et plus généralement aux valeurs de la République, car je considère que les valeurs de la République y sont en danger. Il y a beaucoup d'argent dans certains endroits de notre pays, mais aussi beaucoup de pauvreté en fraternité dans ces mêmes endroits, et beaucoup de nécessiteux en valeurs républicaines. À ce niveau de séparatisme social, et donc de refus de fraternité, le vivre ensemble n'est qu'un leurre, avec des jeunesses qui ne se fréquentent pas, ne se parlent pas. Comment, ensuite, l'élite qui gouverne peutelle comprendre un peuple qu'elle n'a jamais vu de près, pas même pendant sa jeunesse?

Rappelons l'avertissement que donnait Jules Michelet à ses étudiants en 1848, car il me semble bien que cet avertissement est toujours valable aujourd'hui : «Nulle fraternité hors du droit, nul amour dans l'iniquité, nulle alliance hors du cercle que doit tracer la justice $»^{10}$.

\section{L'idée de république fraternelle : une utopie?}

21 A. Perrin-Doucey : dans le cadre de votre mission d'Inspecteur général de l'éducation nationale, vous avez remis en 2015 un rapport qui interroge la corrélation entre grande pauvreté et réussite scolaire et préconise le choix de la solidarité pour la réussite de tous. Comme beaucoup d'enseignants et de formateurs d'enseignants, j'ai lu avec un grand intérêt, teinté d'admiration, ce rapport. En effet, il retranscrit très bien ce à quoi je me suis trouvée confrontée tant dans mon exercice professionnel que dans ma vie militante. Aujourd'hui, pensez-vous que le projet d'une République fraternelle puisse dépasser le stade de l'utopie, que l'école puisse être un levier pour faire vivre la fraternité en éducation?

J.-P. Delahaye : La fraternité peut bien entendu vivre en éducation, et dans beaucoup d'écoles et établissements ce n'est pas une utopie. Je l'ai vu à maintes reprises dans les différentes fonctions que j'ai pu occuper dans mon parcours.

Tréma, 53 | 2020 


\section{Fraternité républicaine et « principe d'éducabilité de tous »}

Les écoles et les établissements qui mettent en œuvre les valeurs républicaines et donc la fraternité sont des établissements dont les pratiques sont conformes aux valeurs proclamées. C'est-à-dire qu'ils promeuvent une éthique collective de l'action pédagogique et éducative autour de quelques principes, comme celui-ci : tous les élèves de l'établissement peuvent réussir et aucun n'est condamné à l'échec : c'est le principe d'éducabilité de tous ; ou encore celui-là : la promotion de tous ne nuit à personne.

Autrement dit, ce sont des équipes qui ont su trouver la force nécessaire pour s'opposer au «sauve qui peut » et au «chacun pour soi » dans l'école ou l'établissement, une stratégie qui profite comme on le sait aux plus favorisés.

A. Perrin-Doucey : quels sont les piliers de cette stratégie?

J.-P. Delahaye : Ce sont des établissements accueillants. Je veux dire par là que ces établissements montrent en permanence aux élèves, quels qu'ils soient, qu'ils sont attendus, qu'ils ont toute leur place dans l'école, le collège, le LP ou le LEGT. Personne ne doit et ne peut se sentir étranger dans un établissement de la République. L'établissement, c'est d'abord un accueil éducatif fraternel.

\section{Une fraternité en actes}

7 Ce sont des établissements justes. Vous le savez, les élèves ont un sens aigu de l'injustice. Et, pour eux, l'injustice commence avec une note mal comprise, une orientation non désirée, une sanction ou une exclusion incompréhensible, une punition collective. Mais un établissement juste n'est pas un établissement laxiste. Les élèves ne s'y trompent pas qui sont souvent les plus sévères en conseil de discipline pour sanctionner les dérapages de certains d'entre eux.

28 Ces établissements sont respectueux et ambitieux. Un établissement scolaire dont tous les membres, adultes et élèves, sont réciproquement respectueux, c'est-à-dire attentifs aux autres, aux personnes et à leurs droits, attentifs aux règles communes, est un établissement fraternel plus à l'abri qu'un autre du désordre et de l'injustice. Un établissement respectueux est aussi un établissement ambitieux pour tous ses élèves.

Ces établissements veillent à la répartition des élèves dans des classes hétérogènes. Leurs moyens sont répartis dans les classes en fonction des besoins : on donne donc plus à ceux qui ont le plus besoin. Les emplois du temps des classes donnent la priorité aux intérêts pédagogiques des élèves, ce qui n'est pas incompatible avec le souci de donner de bonnes conditions de travail aux enseignants. Les équipes veillent à ce que l'organisation des voyages scolaires ne mette pas en difficulté les familles modestes. Les listes de fournitures scolaires sont raisonnables et élaborées collectivement et en concertation avec les parents.

Ces établissements font vivre le principe de co-éducation. Pour garantir la réussite de tous les élèves, ces écoles ou établissements savent associer et faire participer tous les parents, quels qu'ils soient. Ils favorisent une alliance éducative entre tous les partenaires de l'école : parents, collectivités locales, associations.

Enfin, les directions d'école, de circonscription ou d'établissement, de département ou d'académie, la gouvernance donc, mettent en œuvre des méthodes de pilotage collégial 
à tous les niveaux. Que serait un diagnostic de sécurité, un projet d'établissement, un contrat d'objectifs rédigé ou négocié par le seul chef d'établissement sans mobilisation de tout l'établissement?

Une fraternité en actes en quelque sorte.

A. Perrin-Doucey : est-ce du seul ressort des établissements?

\section{Des moyens en cohérence avec l'objectif}

J.-P. Delahaye : Pour que cela ne soit pas une utopie, il faut que les moyens budgétaires soit en cohérence avec l'objectif, sinon la fraternité est une incantation. Certes, il existe des politiques de discrimination positive qui permettent de donner un peu plus à ceux qui ont le plus de besoins. La Cour des Comptes a remis un rapport sur l'éducation prioritaire qui montre qu'en effet: "l'éducation prioritaire mobilise des outils spécifiques qui génèrent un coût global et un surcoût par élève, significativement augmentés par la refondation de la politique engagée en $2014 »^{11}$.

Mais ce « surcoût » est en pratique compensé parce qu'on y trouve des enseignants plus jeunes et souvent non titulaires, donc moins payés qu'ailleurs. A effectifs d'élèves identiques, un collège en éducation prioritaire, malgré les quelques postes supplémentaires dont il bénéficie, peut avoir une masse salariale inférieure à celle d'un collège de centre-ville. La fraternité est ici chichement comptée.

\section{Une fraternité à l'envers}

Prenons un exemple. La dépense pour l'accompagnement éducatif (aide aux devoirs notamment) en éducation prioritaire a été chiffrée par la Cour des comptes à 32 millions d'euros pour 1,7 million d'élèves pour 2016. Cela représente une dépense moyenne de 18,80 euros par élève ! Une évaluation réalisée en 2012 indiquait que l'on dépensait 70 millions pour l'accompagnement les élèves de classes préparatoires, sous la forme d'heures d'interrogation (dites heures de colle) pour préparer les concours. Soit 45 fois plus par élève. Les économies réalisées sur les plus faibles servent ici à préserver les privilèges des plus forts, une fraternité à l'envers en quelque sorte.

Qui sont les assistés dans notre pays? Il est permis de souhaiter que la partie de la population française qui a organisé l'école essentiellement pour ses propres enfants comprenne qu'il est dans son intérêt de mieux organiser l'école pour la réussite de tous. Dans le cas contraire, nous allons au-devant de grandes difficultés. Tant de privilèges, d'un côté, et de scolarités difficiles, d'échecs humiliants, de l'autre, mettent en danger notre pacte républicain. Ceux qui, à tort ou à raison estiment qu'ils n'ont pas accès aux mêmes droits que les autres, auront des difficultés à accepter longtemps d'avoir les mêmes devoirs que les autres.

A. Perrin-Doucey : Une autre répartition des moyens financiers est-elle la seule voie?

J.-P. Delahaye: Les moyens financiers sont nécessaires mais c'est la question pédagogique qui est déterminante. C'est sans doute pour cela que la pédagogie est autant combattue et caricaturée par ceux qui ne veulent rien changer. La haine de la pédagogie manifestée par certains ${ }^{12}$ est en réalité une façon de s'opposer farouchement à un élargissement de la base sociale de la réussite. Bien sûr, il n'existe pas qu'une seule approche pédagogique pour faire réussir tous les élèves, il existe même des approches pédagogiques néfastes qui en rabattent sur les exigences et qui enferment les élèves 
dans leur position d'origine. Mais l'on sait que la solidarité et la coopération ${ }^{13}$ font plus progresser les élèves que le « chacun pour soi » et la compétition.

\section{« Des moments de grâce pédagogique »}

39 Je confirme qu'il ne s'agit pas d'utopie. Ces établissements existent. La fraternité dans un établissement qui pratique la pédagogie coopérative, ce sont les élèves qui en parlent le mieux. Tels ces élèves et ces enseignants du lycée Le Corbusier d'Aubervilliers que j'ai pu entendre lors de leur audition au Conseil économique, social et environnemental (CESE) en 2015 à l'occasion de mon rapport sur la grande pauvreté ${ }^{14}$. Le projet de cet établissement est effectivement basé sur la solidarité et la coopération. Je transcris ici mot pour mot ce qu'a dit un élève de première lors de l'audition de son lycée :

Dans notre lycée, nous avons un projet qui s'appelle «L'Envol », où la solidarité est le mort d'ordre et où il y a une mutualisation du travail. Cela permet à chacun de mieux comprendre les cours et aussi de travailler en groupe, ce qui instaure de la solidarité, et le résultat, en seulement deux trimestres maintenant, c'est une meilleure réussite globale, une meilleure entente entre les élèves et surtout une meilleure compréhension de l'école et des savoirs qui y sont transmis.

Ne pensez-vous pas que la pression, l'évaluation, la saine concurrence, l'esprit de compétition, la réussite matérialisée par les notations, c'est quand même aussi l'apprentissage de ce qui va vous arriver dans peu de temps, à savoir l'apprentissage de la vie sociale et professionnelle ?...Car le monde du travail, c'est la compétition, c'est la notation, c'est l'évaluation, c'est la concurrence et c'est aussi, de temps en temps, beaucoup de plaisir à travailler?

C'est vrai que, dans le monde professionnel de notre société capitaliste, il y a beaucoup de concurrence, surtout entre les employés pour garder leur poste ou même pour avoir un poste. Néanmoins, il me semble aussi qu'il y a des entreprises qui sont basées sur la solidarité. Peut-être est-ce le modèle social de notre société dans sa globalité qu'il faut changer, mais, en tout cas, je soutiens fermement que c'est par la solidarité que l'on parvient à avancer dans les meilleures conditions. En effet, finalement, écraser l'autre, si c'est vraiment cela notre objectif, je ne pense pas qu'on arrive à une atmosphère de travail si saine que cela et je me demande si ce sont ces résultats que l'on veut vraiment obtenir. Si la question est de savoir si la concurrence nous pousse à travailler, peut-être, mais je pense que c'est plutôt la considération pour l'autre qui nous pousse à travailler, et en tout cas, la volonté d'aider l'autre peut aussi nous aider à travailler, et pas seulement la mise en concurrence.

42 Quelle fierté pour les enseignants, et je dois dire pour moi aussi, que d'entendre cet élève donner tout son sens à cette formidable action pédagogique éducative fondée sur la fraternité. Fierté oui, parce que c'est dans ces moments de grâce pédagogique qu'on se sent fier mais aussi honoré d'avoir pour collègues de tels enseignants.

\section{Faire vivre une éducation fraternelle}

A. Perrin-Doucey : Finalement, selon vous, pourquoi est-il nécessaire de faire vivre une éducation fraternelle? 
J.-P. Delahaye: Parce qu'une éducation fraternelle permettra de dépasser la contradiction entre le besoin de solidarité à l'égard des plus faibles et le mouvement de repli qui protège de l'autre. C'est le défi auquel nous devons répondre. Il y va de l'intérêt général car la réussite du plus grand nombre aura des effets bénéfiques sur chacun. Dans une période de crise économique et sociale, la fraternité nécessaire pour la réussite scolaire de tous se heurte inévitablement à des intérêts particuliers qui n'ont pas forcément besoin et donc envie que l'école se transforme.

Et ceux qui s'opposent à la transformation d'un système qui fait si bien, mais à quel prix !, réussir leurs propres enfants se situent sur l'ensemble de l'échiquier politique, savent se faire entendre, défendent les positions acquises, et ont un pouvoir de retardement des réformes, voire de blocage. Une partie de notre problème réside dans le fait que les dysfonctionnements de notre école qui ne parvient pas à réduire les inégalités ne nuisent pas à tout le monde. Globalement, ils ne nuisent pas aux enfants des milieux favorisés, aux enfants d'enseignants, de journalistes, de cadres supérieurs et des élites dirigeantes. Et les bénéficiaires potentiels d'une politique éducative plus fraternelle, notamment les neuf millions de pauvres et leurs enfants, sont, eux, coupés du monde politique et d'une école qui ne sait pas les associer aux choix pédagogiques ; ils n'accèdent pas aux médias et donc ne se font pas entendre. Les milieux populaires ne peuvent donc peser sur les politiques scolaires.

\section{Faire vivre nos valeurs}

Au total, une action éducation plus fraternelle, c'est faire vivre nos valeurs, au moins pour trois raisons.

C'est d'abord une question de justice pour les plus fragiles. Ne sous-estimons pas les dégâts humains et le gâchis immense que représentent des années de scolarité qui ne sont qu'une suite d'échecs et d'humiliations. Tous les enfants des familles pauvres ne sont pas en échec scolaire, mais ceux qui échouent à l'école aujourd'hui seront les exclus de demain. En favorisant davantage la réussite scolaire des plus pauvres, le système éducatif leur offre le seul moyen à long terme de sortir de la détresse.

4 Faire réussir tous les enfants est ensuite une question essentielle pour notre démocratie et notre pacte républicain, car nous ne pourrons indéfiniment prôner le « vivre ensemble » sur le mode incantatoire et dans le même temps abandonner sur le bord du chemin une partie des citoyens. Il y a aujourd'hui parmi les jeunes de 15-29 ans 1,6 million de jeunes femmes et de jeunes hommes qui ne sont ni en emploi ni en formation ${ }^{15}$ et beaucoup d'entre eux sont invisibles du reste de la société car ils ne sont pas non plus en accompagnement. Que font-ils de leurs journées, à qui pensent-ils et, accessoirement, que votent-ils?

L'échec scolaire de trop nombreux enfants des milieux populaires n'est pas seulement un problème pour l'école, c'est aussi une menace pour la République. 


\section{BIBLIOGRAPHY}

Connac, S. (2017). Apprendre par les pédagogies coopératives, préface Jean-Paul Delahaye. Paris : ESF éditeur.

Cosnard, D. (2019). Dans le 16e arrondissement de Paris, la préfecture intervient pour ouvrir un gymnase aux migrants. Le Monde, 28 janvier 2019. https://www.lemonde.fr/politique/article/ 2019/01/28/dans-le-16e-arrondissement-les-migrants-peinent-a-trouverabri_5415803_823448.html

Cour des Comptes. (2018) L'éducation prioritaire. Rapport d'évaluation d'une politique publique. https://www.ccomptes.fr/fr/publications/leducation-prioritaire

Debray, R. (2009). Le moment fraternité. Paris : Gallimard, 2009.

Delahaye, J.-P. (2015). Grande pauvreté et réussite scolaire, le choix de la solidarité pour la réussite de tous. Ministère de l'éducation nationale. https://www.education.gouv.fr/cid88768/grandepauvrete-et-reussite-scolaire-le-choix-de-la-solidarite-pour-la-reussite-de-tous.html.

Finkielkraut, A. (2013). L'identité malheureuse. Paris : Stock.

Le Mitouard, E. (2019). Le centre SDF du bois de Boulogne prolongé jusqu'en 2024 ? Le Parisien, 17 mars 2019. http://www.leparisien.fr/paris-75/le-centre-sdf-du-bois-de-boulogne-prolongejusqu-en-2024-17-03-2019-8033604.php

Fourquet, J. (2018). 1986-2017 : quand les classes favorisées ont fait sécession, Fondation Jean Jaurès, février 2018. https://jean-jaures.org/nos-productions/1985-2017-quand-les-classes-favoriseesont-fait-secession.

Michelet, J. (1970). $8^{\mathrm{e}}$ leçon, 3 février 1848. L'étudiant. Paris : Seuil.

Observatoire National de la Pauvreté et de l'Exclusion Sociale. (2018). Déterminisme social : "L'inégalité des chances en France est d'abord une inégalité des chances éducatives ». http:// www.onpes.gouv.fr/determinisme-social-inegalite-des-chances-educatives.html

Rochex, J.-Y. Crinon, J. (2011) La construction des inégalités scolaires. Au cœur des politiques et des dispositifs d'enseignement. Rennes : Presses Universitaires de Rennes.

Saint-Exupéry A. (1982). Ecrits de guerre 1939-1944. Paris : Gallimard.

Wagnon, S. (2019). Pour une pédagogie solidaire. Avant-propos de Francis Vallat, préface de Philippe Meirieu, postface de Jean-Paul Delahaye. Aniche : Sipayat éditions.

\section{NOTES}

1. Dorénavant ONPES.

2. Observatoire National de la Pauvreté et de l'Exclusion Sociale. (2018). Déterminisme social : « L'inégalité des chances en France est d'abord une inégalité des chances éducatives ». http:// www.onpes.gouv.fr/determinisme-social-inegalite-des-chances-educatives.html

3. https://www.education.gouv.fr/cid88768/grande-pauvrete-et-reussite-scolaire-le-choix-de-lasolidarite-pour-la-reussite-de-tous.html

4. Saint-Exupéry A. (1982). Ecrits de guerre 1939-1944. Paris : Gallimard.

5. CNESCO, Mixité sociale à l'école, juin 2015. http://www.cnesco.fr/fr/mixites-sociales/ 
6. Voir Jérôme Fourquet, 1986-2017 : quand les classes favorisées ont fait sécession, Fondation Jean Jaurès, février 2018. https://jean-jaures.org/nos-productions/1985-2017-quand-les-classesfavorisees-ont-fait-secession

7. Régis Debray, Le moment fraternité, Gallimard, 2009, p. 11.

8. NDLR «Ici, Jean-Paul Delahaye évoque l'opposition violente au projet, proposé en 2016 par la mairie de Paris et l'association Aurore, d'installation d'un centre d'hébergement d'urgence pour sans-abri, allée des fortifications, en lisière du Bois de Boulogne, dans le $16^{\text {ème }}$ arrondissement. Une réunion d'information des riverains réunissant plus de 1000 personnes a, selon la Presse, été interrompue violemment par les participants hostiles au centre. Une pétition contre le projet a réuni plus de 40000 signatures. Finalement, les opposants n'obtiennent pas gain de cause et le projet est autorisé à titre provisoire pour 3 ans. A l'automne 2019, alors que le centre provisoire comme la scolarisation des enfants ne posent « aucun problème » (Le Parisien, 17 mars 2019), la maire LR de l'arrondissement s'oppose à la prolongation du permis de construire, par principe affirmant que "trois ans, c'est trois ans par une année de plus». Ajoutons que d'autres contestations font rage. En janvier 2019, la Préfecture doit intervenir "pour permettre de transformer comme prévu [une] salle de sports en hébergement d'urgence pour les migrants dans le cadre du plan grand froid, à titre "très provisoire » ». (Le Monde, 28 janvier 2019). Cette fois encore les riverains s'opposent, en plein hiver, à un accueil provisoire des plus nécessiteux. » 9. NDLR « Rappelons que cette expression de "territoires perdus de la République" renvoie au titre d'un ouvrage collectif dirigé par G. Bensoussan paru en 2002 aux éditions Mille et une Nuits : Les Territoires perdus de la République - antisémitisme, racisme et sexisme en milieu scolaire. Réédité en livre de Poche en 2015, l'ouvrage, qui rassemble des témoignages d'enseignants et de chefs d'établissements, vise à dénoncer la montée des discriminations qui s'expriment violemment dans les établissements scolaires de banlieue. Luc Ferry, alors ministre de l'éducation nationale, puis Alain Finkielkraut ont tour à tour popularisé l'expression pour dénoncer (ou stigmatiser) « la triste réalité des quartiers difficiles » (Finkielkraut A. (2013)

10. Jules Michelet, $8^{\mathrm{e}}$ leçon, 3 février 1848, dans L'étudiant, Paris, Seuil, 1970, p. 137.

11. Cour des Comptes, L'éducation prioritaire, Rapport d'évaluation d'une politique publique 2018. https://www.ccomptes.fr/fr/publications/leducation-prioritaire

12. NDLR «Rappelons ici les violentes diatribes lancées, notamment au moment de chaque élection présidentielle contre le pédagogisme, néologisme destiné à fustiger les méthodes d'apprentissage liées historiquement aux mouvements d'éducation nouvelle et qui souhaitent notamment faire des élèves des acteurs de leurs apprentissages. "

13. Voir notamment Jean-Yves Rochex, Jacques Crinon, La construction des inégalités scolaires. Au cœur des politiques et des dispositifs d'enseignement, PUR, 2011. Sylvain Connac, Apprendre par les pédagogies coopératives, préface Jean-Paul Delahaye, ESF éditions, 2017. Sylvain Wagnon, Pour une pédagogie solidaire, Avant-propos de Francis Vallat, préface de Philippe Meirieu, postface de JeanPaul Delahaye, Sipayat éditions, 2019.

14. Jean-Paul Delahaye, Grande pauvreté et réussite scolaire, le choix de la solidarité pour la réussite de tous, Ministère de l'éducation nationale, 2015. https://www.education.gouv.fr/cid88768/grandepauvrete-et-reussite-scolaire-le-choix-de-la-solidarite-pour-la-reussite-de-tous.html

15. Insee Références, édition 2018 - Fiches - L’insertion des jeunes. 


\section{AUTHORS}

AGNÈS PERRIN-DOUCEY

Université de Montpellier, LIRDEF

JEAN-PAUL DELAHAYE

Inspecteur général de l'éducation nationale honoraire 